\title{
CONNEXIONS: DSP EDUCATION FOR A NETWORKED WORLD
}

\author{
R. G. Baraniuk, C. S. Burrus, B. M. Hendricks, G. L. Henry, A. O. Hero III, D. H. Johnson \\ D. L. Jones, J. Kusuma, R. D. Nowak, J. E. Odegard, L. C. Potter, K. Ramchandran \\ R. J. Reedstrom, P. Schniter, I. W. Selesnick, D. B. Williams, W. L. Wilson
}

cnx.rice.edu

\begin{abstract}
Connexions is a new approach to authoring, teaching, and learning that aims to fully exploit modern information technology. Available free of charge to anyone under open-content and open-source licenses, Connexions offers custom-tailored, current course material, is adaptable to a wide range of learning styles, and encourages students to explore the links among courses and disciplines. In contrast to the traditional process of textbook writing and publishing, Connexions fosters world-wide, cross-institution communities of authors, instructors, and students, who collaboratively and dynamically fashion "modules" from which courses are constructed. We believe the ideas and philosophy embodied by Connexions have the potential to change the very nature of textbook writing and publishing, producing a dynamic, interconnected educational environment that is pedagogically sound, both time and cost efficient, and fun. This paper overviews the philosophy and technology behind Connexions and describes a nascent community developing material for DSP education.
\end{abstract}

\section{INTRODUCTION}

The lynch-pin of university teaching has traditionally been the textbook. Unfortunately for students, teachers, and authors, textbooks - whether in paper form or on the web - suffer from severe shortcomings that impede the education process.

Sequential and inflexible: All paper and most web-based textbooks cover topics sequentially, which both misleads and bores students. Their self-contained nature discourages holistic curriculum development by obscuring the connections among the range of courses that make up a discipline. Further, existing texts provide inflexible, off-the-rack content that caters to only a single learning style and is often "not quite right" for the instructor's needs.

Static and inefficient: The current development model for textbooks is static and extremely inefficient. At present, texts are developed by a single author or small team and take years to write, reach bookstores, and undergo revision. Only a very small proportion of potential authors ever contribute to the body of published materials due to this large time commitment.

Connexions is an experimental approach to authoring, teaching, and learning that aims to fully exploit the flexible information organization and rapid communication capabilities of the Internet and World Wide Web. Born at Rice University in 1999, the project has now begun to expand globally. Based on our promising preliminary results, we believe that Connexions has the potential to set the standard for the development, organization, and delivery of educational materials in the networked age.

In a nutshell, the Connexions concept can be explained using the "factory" analogy of Figure 1. A global community of

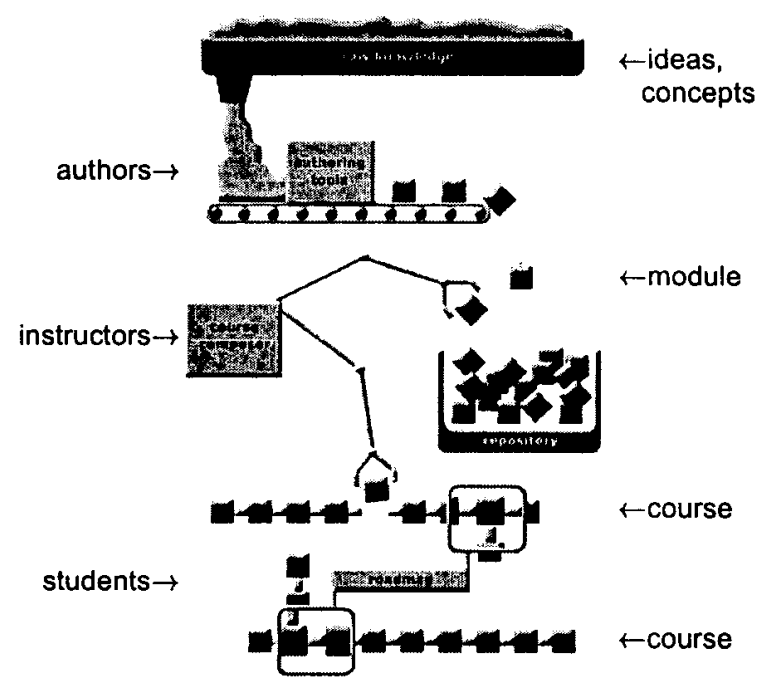

Fig. 1. Connexions as a "course factory."

authors continuously converts "raw knowledge" into small, selfcontained modules of information, the equivalent of a page or two in a textbook. Modules can be imagined as special web pages with hyperlinks pointing to prerequisites, applications, and supplementary material. Modules are placed in a common repository to be used, reused, updated, and adapted. Instructors use a Course Composer software tool to weave modules into customized courses that can be placed on the web, presented in class, or printed as a paper text. Students and other leamers access web courses or the repository directly using special navigational tools designed to highlight the non-linear "connexions" among concepts both within the same course and across courses and disciplines. To promote the broadest impact, freest exchange of ideas, and most rapid and dynamic development, all materials are available free of charge under an open-content license $e^{l}$ analogous to the open-source license of the Linux operating system. Likewise, all software tools are opensource. The result is a coherent system for course development, organization, and delivery that mutually benefits students, instructors, and authors.

Connexions' software and content development processes have

'An open-content license allows anyone to read, copy, modify, and redistribute the work as long as they permit others to do the same. 


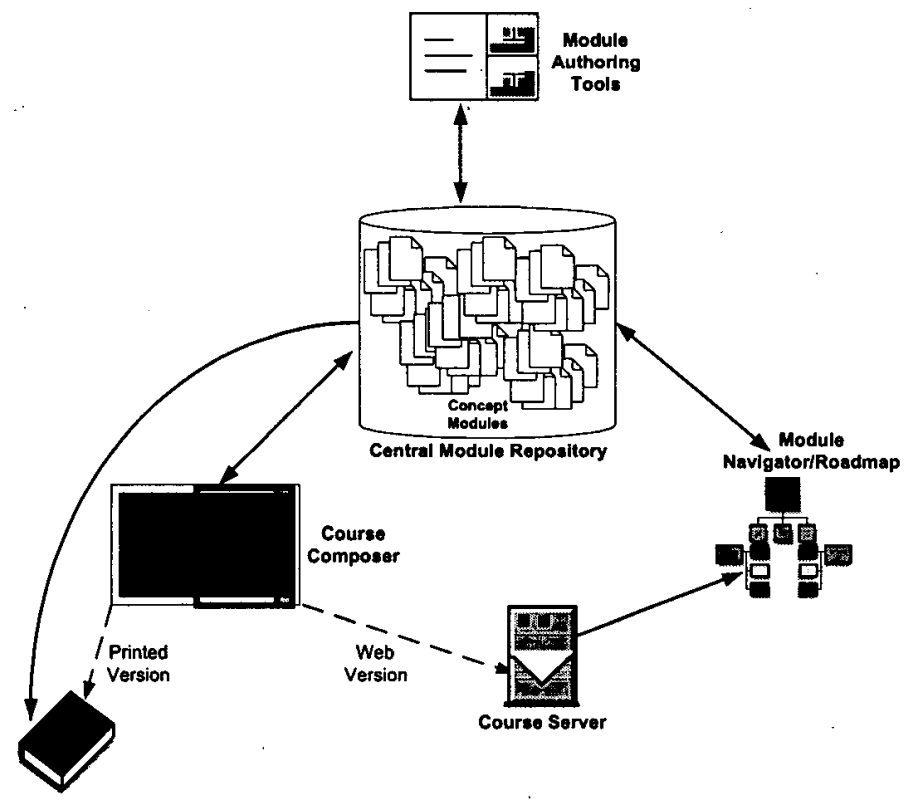

Fig. 2. Connexions architecture and tools.

been carefully designed to go far beyond existing web-based hypertext systems. A number of unique advantages result.

Collaborative and high quality: Connexions fosters the development of a diverse worldwide community of authors who work collaboratively to create, expand, review, and maintain modules and courses. Inherently inclusive, this community can include university faculty, industry professionals, and even talented students. Thanks to peer-review and pride-of-authorship (as in the Linux development model), high-quality modules and courses result.

Efficient and dynamic: Breaking course materials into discrete modules drastically reduces the time commitment from authors and instructors, who can now write a high-quality module or weave a customized course in an evening or weekend. The same module can be reused in myriad different courses and rapidly adapted to new settings. Making all content modules and software freely available (open-content and open-source) leverages the efforts of a worldwide community of scholars and ensures the broadest application of all Connexions materials and tools. The Connexions repository is thus a dynamic, living ocean of knowledge modules that are constantly improving in an evolutionary fashion,

Coherent: All Connexions modules are encoded in a single coherent format that allows them to be woven together seamlessly in infinite different ways and then presented in myriad different output formats, from web pages to e-books to print. Connexions' XML format also encodes and displays integrated mathematical equations, chemistry formulae, musical notation, and so on. The seamless reusability of Connexions modules lies in stark contrast to the hodge podge of loosely connected HTML, PostScript, PDF, Microsoft Word, and Power Point documents comprising the world's many educational web sites.

Holistic and diverse: By linking concepts across courses and even disciplines, Connexions provides students with a "big picture" vision that no traditional textbook can provide. Moreover, a great diversity of ideas, ideologies, and pedagogical methodologies can be easily and quickly introduced through modular course construction. Connexions helps instructors build curricula by bringing into focus the gaps between courses and by making it simple to provide both prerequisite and advanced knowledge. Students less prepared for courses can overcome deficits by studying modules on prerequisite topics. Well-prepared students ready for advanced knowledge and skills can pursue broader interests, accelerate their acquisition of core knowledge, and develop advanced research and design skills.

Flexible and current: Modules can be developed to cover a wide range of learning styles and can be translated into many different languages. Materials can be developed across many fields of study, and they can be easily adapted to, and developed in, a variety of educational environments, including $\mathrm{K}-12$, continuing, distance, self-paced, and corporate education. Since modules can be easily added and adapted, their information stays current.

This paper overviews the Connexions architecture and tools, our experimental DSP content community, and our plans for the future.

\section{CONNEXIONS ARCHITECTURE AND TOOLS}

The Connexions architecture and tools sketched in Figure 2 echo the factory analogy of Figure 1. The tools are designed to be flexible, scalable, and easy to use for authors, instructors, and students alike [1]. Alpha versions are currently in live use at Rice University and by faculty at several other institutions.

Content modules: Authors compose modules using the extensible markup language (XML) [2], which allows Connexions to exhibit the links between related modules, support myriad output formats, enable powerful search mechanisms, and display and print clear and attractive mathematics (using MathML). XML generalizes the familiar HTML that forms the backbone of the World 
Wide Web. Connexions' specifically designed markup language for course content is called $c n x M L$. Since XML encodes what the content means rather than how it should be presented (displayed), ${ }^{2}$ modules are very flexible - the same cnxML source module can be displayed as an individual web page, woven seamlessly into many different courses, converted to LaTeX/PostScript or PDF for printing, or even processed through a speech synthesizer to read material to the blind. The ultimate presentation of a module depends on a style sheet that is chosen by the end-user and not fixed a priori by the author.

Authoring tools: Any of a variety of XML and MathML editors can be used to generate new modules. Most of these editors remain primitive at present, making content input more tedious than, say, writing in LaTeX. Content conversion from standard formats (such as LaTeX, Word, or Power Point) is complicated by the fact that these formats are primarily presentation based rather than content based. Consequently, developing an easy-to-use authoring suite is now our software team's highest priority. Fortunately for busy faculty authors, the excitement surrounding XML and web development facilitates recruiting students to aid with content input. The Connexions author web site enables authors to communicate with each other, collaborate on shared modules in ad hoc workgroups, and submit their work to the repository.

Module repository: The module repository stores the content modules and manages their access. At present, the repository is a central, rather than distributed, storage system to simplify its maintainability, availability, and reliability. Connexions proxy cache servers are planned for improved access to frequently used modules. Content cnxML modules are stored as text in a version control system (CVS) to maintain their complete revision history, ${ }^{3}$ with the metadata stored in a database for easy search and retrieval. The metadata includes module title, authors, keywords, and the linking structure (which modules link to which other modules).

Course Composer: This tool allows instructors to search the repository for modules, group them into "chapters," sequence them into a course, and add customizing annotations ("margin notes") and segues. The course path and materials can be stored on the Connexions web site or a local Web server and presented through a web browser. Alternatively, a linear PostScript or PDF version can be created for printing locally or spooling to an on-demand publisher such as Kinkos.

Module navigator / Roadmap: The Roadmap tool is a web browser plug-in that guides students through each course, helps them visualize the non-linear connexions between the concepts in

\footnotetext{
${ }^{2}$ HTML, on the contrary, mixes content and presentation information. For example, the HTML <bolds tag finds use around both the journa volume number in an academic citation (as in "SIAM Rev., 34(7)") and shouted words in a theatre piece (as in "Turn that music down!!"). Clearly the HTML <bold > tag carries much presentation and little content information. XML separates content and presentation [2]. In XML, we can define a $<$ volumeNumber $>$ tag for journal citations and a $<$ shout $>$ tag for plays. Furthermore, the end-user can decide, using a "style sheet" how a <shout >'ed item should be displayed; some users may want to italicize shouting (as in "Turn that music down!?"). Similarly, for mathematics, content MathML allows us to encode that the symbol $x(t)$ means "the function $x$ of variable $t$ " rather than "the letter $x$ followed by the symbol (, the letter $t$, and the symbol ), which is how $x(t)$ would be encoded in LaTeX, Word, or Power Point. Because of this extra meaning information, content MathML formulas can be pasted directly into Mathematica, for example, to work as a "living applet."

${ }^{3}$ Version control software is crucial to track changes to a module, to attribute who changed what, and to lock in a specific version for a given course so that it does not change under an instructor's nose.
}

different modules, courses, and curricula, and encourages them to explore related topics and then return easily to their course path Students will be able to make their own personal "margin note" annotations that will be archived permanently on the system. For rapid prototyping, the Roadmap is currently based around Mozilla [3], the open-source web browser for forms the core of Netscape 6. Netscape 6 and Microsoft Internet Explorer do not yet display MathML, but are expected to in the near future.

All Connexions software and tools will be open-source, free of charge, and available soon in beta versions. More information is available at the project website.

\section{CONNEXIONS CONTENT}

The open-content development model is the most radical aspect of Connexions, but it holds the key to populating the repository with a critical mass of high-quality content.

\subsection{Open-content, community development}

Rather than the traditional one-author-to-one-textbook content development model, Connexions links worldwide communities of authors to collaboratively create, expand, revise, and maintain modules and courses. All materials will be freely available under a license analogous to that used for Linux software. The result is a dynamic, up-to-date content base that makes the latest knowledge globally available. Pride of ownership and an inherent peer review system will ensure high quality (more on this below).

Moreover, open development is extremely cost-effective (since no money changes hands) and time-efficient (since it leverages the efforts of a global community and allows each module to be reused in numerous different contexts). Connexions substantially lowers the barrier to entry into the author community. Since authors can now contribute a high-quality, high-impact module in an evening or weekend, many more faculty (and even talented students) will contribute materials. Consider this quote from an electrical engineering faculty member: "For years I have wanted to write a DSP textbook, because I love to write about FFTs. However, any complete DSP text would also have to cover filter design and $z$-transforms, on which I have no interest in writing." Connexions will allow this faculty member to contribute their (excellent) FFT material and then weave a custom text for their DSP course using contributions from other authors passionate about filters and $z$-transforms.

We envision that in the future module usage history (the number of courses it appears in or the total number of visits, for example) and ratings (by an external body such as the IEEE, for example) will become indicators of "teaching contribution" for faculty much as journal paper citations and external reviews are used to measure "research contributions."

\subsection{Content quality control}

Given that anyone can contribute to the Connexions repository and can modify and redistribute modules, how do we ensure that highquality content is easily accessible to users? This requires both a means to evaluate and credential modules and a means to direct users to modules deemed of high quality.

Prereview: Traditional publishers - including the IEEE employ a peer review process. Each author submits a contribution to an editor who, based on reviews by the author's peers, decides

\footnotetext{
${ }^{4}$ Readers unfamiliar with the power of open development should read Eric Raymond's classic essay. "The Cathedral and the Bazaar" [4]
} 
whether to publish the work or not. Only then is the work publicly accessible. Any prereview procedure is too unwieldy to keep up with the fast pace of Connexions module updates, where a module may change daily or even hourly. Moreover, the traditional binary decision to accept/reject a work is inappropriate when a module can improve in an evolutionary fashion. Accept/reject decisions also create an exclusive rather than inclusive community culture. Thus, all content submitted to Connexions will be accessible from its repository. Nevertheless, Connexions' success depends on users having quick and simple access to high-quality content.

Postreview: Rather than make a single prereview yes/no decision regarding each module, Connexions opens up the editorial process to third-party reviewers and editorial bodies for postreview. While Connexions users will have access to all modules (whatever their quality), users will also have the ability to preferentially locate and view modules and courses rated high quality by using a number of different filters provided by third parties. As a simple example of a filter, imagine a professional society independent of Connexions (the IEEE, for example) that sets up a web page containing a list of all Connexions modules and courses that it deems high quality. It can also post reviews of those modules and courses. The list would prove indispensable to students and instructors who trust the opinions of that society. Indeed, users will be able to set up their Roadmap browser to view preferentially those modules approved by the editorial bodies of their choice. ${ }^{5}$ of course, users will always have the option of turning off all filtering to view the repository in its entirety.

\subsection{DSP content project}

Faculty at Rice University have embraced the Connexions approach to authoring, and their contributions have been limited only by our ability to feed their material into the system. Over five hundred modules now form the basis for five electrical and computer engineering undergraduate courses at Rice as well as the framework for a holistic new information technology curriculum spanning the departments of electrical and computer engineering, computer science, and applied mathematics.

In a Connexions workshop in August 2001, faculty members from the Georgia Institute of Technology (D. Williams), Ohio State University (L. Potter, P. Schniter), MIT/Berkeley (J. Kusuma, K. Ramchandran), Polytechnic University (I. Selesnick), Rice University (R. Baraniuk, C. S. Burrus, D. Johnson, R. Nowak), University of Illinois (D. Jones), and University of Michigan (A Hero), formed an author/instructor "beta community" that aims to develop several hundred modules for teaching graduate-level digital signal processing (DSP).

DSP education is a ripe opportunity for Connexions to illustrate the community-building process. With the rousing commercial success of DSP, many graduate textbooks have been migrated to the undergraduate level. The breadth of the field complicates graduate textbook development, as each school teaches widely ranging topics; one single text covering most topics would contain several thousand pages. Nevertheless, there exist many commonalities between the courses at various institutions. The Connexions

\footnotetext{
5Example: "While I search and navigate Connexions, preferentially display those modules and courses rated four stars or higher by the IEEE Signal Processing Society or those used in courses at Caltech or ENSTParis." Other possible filtering criteria include "most visited module on a certain topic," "recommended by a trusted colleague," and "recommended by 'similar' users" (as Amazon.com suggests books).
}

DSP community aims to develop a common content base from which a customized DSP course can be woven for each school. DSP laboratories are also being developed, since the same theoretical background and many algorithmic details can be shared among laboratories based on different DSP chip architectures.

Current content development focuses on the following themes: (1) signal spaces, linear algebra, and least squares theory; (2) random processes, detection, and estimation; (3) wavelets, filterbanks, multirate, and source coding; (4) spectrum estimation; and (5) adaptive filtering. Content input ranges from existing handwritten and LaTeX class notes to partially completed textbooks to completely new material.

\section{SUMMARY AND ISSUES}

"Boundless" is a particularly appropriate adjective to summarize Connexions: by freeing ideas and concepts from languishing bound in traditional books, we have created a new approach to developing and delivering educational content that is truly boundless in scope.

Our overarching goal is to take Connexions to critical mass on a global scale. Our development plan consists of three inter-related thrusts: (1) Content: Launch several pioneering open-content development projects in several diverse subject areas, in addition to the aforementioned one in DSP, and develop and experiment with holistic Connexions curricula. (2) Software: Develop a scalable software infrastructure to support collaboration among authors, instructors, and students on a global scale. (3) Organization: Launch Connexions as a non-profit, "dot-org" that is independent of any university or publisher to best serve its global communities.

Interesting issues abound, including (1) the legal subtleties surrounding how faculty can contribute and use Connexions material; (2) the rôle commercial publishers can play in adding value to open-licensed Connexions material, analogous to how for-profit companies such as Red Hat and IBM support the development of Linux; (3) the interplay with other related projects, including MIT's Open Course Ware (OCW) project (web.mit .edu/ocw) and the Open Knowledge Initiative (OKI) (web . mit . edu/oki).

One particularly interesting challenge we already face in the DSP content project involves mathematical notation. In the first generation of the material, we are agreeing upon a standard set of notation. Meanwhile, a Connexions technical team plans to develop a set of standard MathML "DSP macros" that can be displayed according to the end-user's taste in notation. For example, the macro <dtimesignal > could be displayed as $x(n), x[n]$, $x[m], s[n]$, etc. Again, this separation of "meaning" from "presentation" will greatly facilitate fusing material from many different authors, each of whom has a different notational taste.

To learn more about Connexions or to get involved as an author, instructor, student, or software developer, visit the project website at cnx. rice.edu or email cnx@rice. edu.

\section{REFERENCES}

[1] B. M. Hendricks, Connexions: An Architecture for Webbased Educational Materials, M.Sc. Thesis, ECE Dept., Rice University, Jan. 2001

[2] XML, w3c.org.

[3] Mozilla, mozilla.org.

[4] E. S. Raymond, The Cathedral and the Bazaar, www. tuxedo.org/ esr/writings/cathedralbazaar. 